\title{
From Toys to Games: Overcoming the View of Natural Selection as a Filter
}

Víctor J. Luque Cavanilles Institute of Biodiversity and Evolutionary Universitat de València victor.luque@uv.es

DOI 10.1515/kjps-2016-0014

\section{Filters and Toys}

The conceptualization of natural selection by Elliott Sober has had an enormous influence on both philosophers of biology and professional biologists'. He addresses, in his work The Nature of Selection ${ }^{2}$, the issues of how to understand and explain natural selection. This position has become known as the 'Negative View'. It maintains that natural selection is a negative force or cause which works as a filter by eliminating those individuals with less fit traits and leaving the rest intact. From this view, natural selection has only a distributive role: the existence of population variation is assumed and selection only changes traits frequencies in it ${ }^{3}$. In this case, Sober follows a long-standing tradition that dates back to

\footnotetext{
$1 \quad$ For example see Endler, 1986; and Futuyma, 2013.

2 Sober, 1984.

3 Godfrey-Smith, 2009.
} 
Hugo de Vries ${ }^{4}$ and other authors (Morgan, Punnet) from the early twentieth century. This position denies that selection is able to explain the origin of a trait or, as De Vries claimed, "Natural selection may explain the survival of the fittest, but it cannot explain the arrival of the fittest"5.

In opposition to the Negative View we find the 'Creative View' 6 - whose origins can be traced back to Darwin ${ }^{7}$ himself. From this view, selection has a positive or creative role, acting not only by eliminating variants but creating the best variants; not only explaining the survival of the fittest but also creating the fittest ${ }^{8}$. Sober denies such ability by pointing out a distinction which he attributes to Darwin himself, i.e., the difference between variational and developmental explanation. Sober affirms Darwin did not give a different explanation to an old problem - adaptation when he developed the concept of natural selection, but he created a new kind of explanation. Following on from Lewontin ${ }^{9}$, Sober defends that before Darwin - that's, for example, Lamarck's case - the explanation of a biological phenomenon was based on an organisms' development. "In contrast, Darwin's theory of evolution of species is not developmental. Darwin explained change in a species by a mechanism that permits (and, in a sense to be made clear, even requires) stasis in organism"10. Thus, developmental explanation appeals to the development of the individual trait, to its obtaining. However, variational explanation appeals not to the individual level but to the population level. Sober uses a widely quoted example to explain it: Imagine a class where all children read at a third grade level - and those who cannot read at a third grade level would not belong to this class. Its different components (Sam, Aaron, Marisa, etc.)

$4 \quad$ "Natural selection acts as a sieve; it does not single out the best variations, but it simply destroys the larger number of those which are, from some cause or another, unfit for their present environment" (De Vries, 1909, 68).

5 De Vries, 1904, 825-826.

6 Nanay, 2005; Martínez and Moya, 2011.

7 "What limit can be put to this power, acting during long ages and rigidly scrutinising the whole constitution, structure, and habits of each creature, favouring the good and rejecting the bad? I can see no limit to this power, in slowly and beautifully adapting each form to the most complex relations of life" (Darwin, 1859, 469).

8 Gould, 2002.

9 Lewontin, 1983.

10 Sober, 1984, 149 (emphasis in the original). 
have the ability to read at that third level. How have they obtained such a reading level? The developmental explanation appeals to the individual development of this ability, i.e. how the students have acquired their skill over the years showing their apprenticeship - how Sam's father helped him with his first reading; how Aaron's teacher taught him to spell; how Marisa enjoyed reading by herself Where the Wild Things Are; etc. Nevertheless, a variational explanation does not need to appeal to the development of individuals' reading ability in order to explain why they can read at third grade level. Individuals, in this case, do not change. They can read at third grade level before and after they come to the class ${ }^{11}$.

Two characteristics of Sober's approach must be emphasized: (i) selection is an explanation which involves elimination or a filter (the reading class admits only certain types of children, consequently some of them come in and some others do not); (ii) selection does not change individuals, they remain static (children continue reading at a third grade level before and after they come to the class). These characteristics are important because they are connected (and supported) by another conceptual novelty developed by Sober: the difference between selection-for and selection-of, and its visual correlation through the selection toy.

\subsection{Sober's selection toy}

Evolutionary biologists, among other tasks, seek to determine which traits have been favoured by natural selection, i.e., those which had a causal role in the survival and reproduction of the individual. In other words, evolutionary biologists have to deal with the problem of distinguishing between direct and indirect selection, between a trait which is

\footnotetext{
11 Birch (2012) has recently challenged, very convincingly, this argument defended by the negative view of natural selection. Negative viewers supported this argument by appealing to a counterfactual criterion $-C$ rather than $C^{*}$ helps explain $E$ rather than $E^{*}$ only if: (if $C^{*}$ had occurred, then $E^{*}$ would have occurred) - where natural selection fails to accomplish it, and therefore lacks of explanatory relevance in order to explain the possession of traits of a particular individual. Birch points out that this criterion is defective because it fails recognizing causes that help to overdetermine the non-occurrence of $E^{*}$. Therefore, Birch proposes a better relevance criterion, based on Strevens' work. This new criterion is able to overcome those problems, and show that the action of natural selection is explanatory relevant in order to explain the possession of traits of a particular individual, vindicating the positive view.
} 
being selected because it is a causal part of the differences in fitness among organisms, and another one which is simply correlated ${ }^{12}$.

Though, this task is not as simple as it seems since sometimes traits are linked to other ones (pleiotropy), it means their evolutionary path befalls simultaneously and in parallel. Nonetheless, it is possible that only one of these traits had been selected because of an individual's survival and reproduction properties, and the other had not ${ }^{13}$. Sober's selection toy helps us to understand the problem. The toy is divided into four platforms with holes of different widths in each of them (from the narrower on the base to the wider on the top). There are balls of different sizes and colours, inside the toy. If all the balls are at the higher platform and we shake the toy, what will happen? Only the smallest balls reach the lowest level of the toy. They are also the green ones. So, the small size and the green colour have been selected, but why have just these balls fallen? The cause is their size, not their green colour. In other words, that's selection for size, and the green colour is only a correlated trait. In short, selection-for size and selection-of colour. For this reason, Sober argued that the overall fitness of an organism - the collection of traits of a particular organism - is not causally efficacious because only some organism's traits may play a role in its survival and reproduction. Therefore, like life expectancy is a summary of possible factors that affect one's life span, organism' overall fitness is a summary of possible factors that affect organism's reproductive success (I return to this topic in section 3.3.).

The selection toy shares with the example of the reading class the two characteristics mentioned above: (i) a filter process where some of the balls remain at the top and some others fall to the bottom (which balls will pass to the next generation); and (ii) balls do not change in the process since the filter keeps them identical. Thus, two conceptual distinctions, variational and developmental explanations and selection-for/ selection-of, support each other in Sober's argumentation. Possibly, this

12 Conner and Hartl, 2004.

13 Distinguishing between direct and indirect selection is a necessary condition for testing adaptationist hypotheses. Therefore statistical multivariate methods have been developed (Lande and Arnold, 1983; Fairbairn and Reeve, 2001). See note 23. 
bidirectional role between them has helped Sober's Negative View to be as persuasive as it is convincing.

Nevertheless, this filtering image faces counterexamples. Imagine a small group of birds (for example, five males and five females), healthy (viable and fertile), which arrive at an island with abundant resources. Its fertility also allows them to find a partner whenever they want. Suppose that a couple from the original group has the ability to metabolize food twice as fast, increasing, consequently, their time to procreate, search for food, etc. The genes from this couple will spread faster than the rest. Nonetheless, the other individuals also leave offspring because of the abundant island's resources and it is not until many generations later that the environment will not be able to support the population size ${ }^{14}$.

In this example, Sober's Negative View based on the image of a filter is unable to perform its explanatory task. The selection toy is based on two principles: (1) Natural selection is (works as) a filter; and (2) it can differentiate between selection-for and selection-of. Now, regarding (1), my example shows that the selection process is not always comparable to the action of a filter. If we look closely, when the group of birds arrive to the island, in that favourable environment (abundance of resources), the population increases over generations ${ }^{15}$. No trait is left behind, no individual dies before the age of childbearing, no couple is infertile, etc. This process is not like a filter. By definition, a filter is a device which removes or selects impurities by blocking their way while, at the same time, allowing the rest to pass through (or vice versa, like gold miners; see section 3). But a filter which allows everything to pass through is not a filter at all.

14 This example is less hypothetical than it may seem. The arrival of a small group of individuals to an island can have huge evolutionary consequences (Vervust et al., 2007).

15 This is an idealized approximation in which we ignore genetic drift as a possible cause of genetic loss. First, the use of ideal models is common in science, both in Physics (frictionless plans, perfect pendulums, etc.) and Biology (infinite populations, no migration, etc.). Second, it is true that in my example drift should be an important causal factor because the smaller the population is, the greater is its strength (Gillespie, 2004), but it would be irrelevant to the purpose of the example because if variability is lost by drift, selection would have had no role in it. That is, the genetic loss will not be caused by a filtering process due to selection. 
On the other hand, the image of filter that the selection toy represents lets Sober differentiate between selection-of and selection-for, between selection of green balls and selection for smallness. But in the birds example there is no way to differentiate between selection-of and selection-for using a filtering view. It is as if all the balls of different colours and sizes have reached the bottom of the toy. Nevertheless, it is obvious that there is selection for fast metabolism. Individuals with this trait increase their frequency compared to those which are normal type. But those do not die either nor decrease their absolute number (in fact, it increases). None of the two characteristics of the selection toy are accomplished: selection (the toy) does not filter any ball (individual or type) because all of them reach the bottom of the toy (the traits of the individuals pass to the next generation); and the toy is unable to differentiate between those properties which have been selected due to their causal role (selection-for) and those which are simply correlated or have been indirectly selected (selection-of) because all of them pass through.

\section{The Selection Game}

Sober's selection toy is insufficient when we want to include complexity within the representation of selection. Maybe we should go a step further and, as children who leave behind their toys and become interested in more intricate games like chess, find a new game model to represent natural selection. My proposal is based on experimental biology carried out with microorganisms (viruses, bacteria and yeast), which have proved to be very useful in the study of evolutionary dynamics and the test of theoretical hypotheses ${ }^{16}$. In this area, natural selection operates when two different types of the same organism are placed in a controlled environment (for example, a culture tube), which is rich in resources. Both types will grow exponentially, leading to different rates of replication. The main element of this approach, and which does not exist in Sober's selection toy, is replication. Replication is one of the basic building blocks of evolution ${ }^{17}$ and my approach to natural selection takes it into account.

16 Elena and Lenski, 2003.

17 Nowak, 2006. 
We can find something like it in Go. Much more widespread in Asia than in Europe, Go is a strategy game based on a kind of replication. Both players try to achieve the highest number of playing pieces (called stones) on the board. Likewise, a selection process requires at least two types that differ in fitness - consequently one of them will expand its traits faster in the population -, much as a particular colour on the Go board will prevail upon the other. In the Asian game alternate turns allow players to add a playing piece each time. But, what would happen if one of the players can add two stones in each of their turns? Our selection game explores that possibility and shows it in a virtual way through an animation which, as a 2.0 version of the analogic Sober's toy, expects to expand its reach and overcome its limitations.

There are two different types of balls in the game. They are the same size but different colour, blue and red, placed in an environment without any spatial restriction beyond the boundaries of the screen (Figure $1 \mathrm{a}$ ). Both types of balls lack mortality and suffer from an exponential increase of their number in order to fill the screen. It is as if we have installed an automatic program of Go. The game establishes a different replication rate to each ball. Thus, the blue ball reproduces two exact copies of itself in each generation, meanwhile the red ball can produce just one copy of itself in each generation. At the beginning of the game we observe that blue balls quickly fill more screen space than red balls, increasing its frequency (Figure 1b). After seven generations, blue balls have almost entirely filled the screen, except for a few red balls in a corner.

Despite no ball 'dying' during the game, there is evolution by natural selection because the game accomplishes the three classical features ${ }^{18}$ : variation (different colour balls); differences in fitness (some of them multiply themselves more than the others due to their colour); and heredity (the colour is transmitted to the next generation of balls). Nevertheless, none of the balls disappear but they multiply until they completely fill the screen. In other words, there is selection without elimination or filtering. Selection begins with the first replication and was maintained during the rest of the generations. The game also distinguishes between

18 Lewontin, 1970. 


\begin{tabular}{l}
\hline \\
\hline
\end{tabular}

\section{Figure 1a}

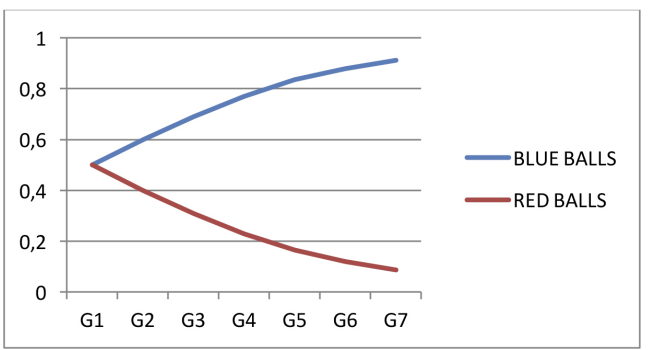

Figure 1b 
selection-for and selection-of since there is selection for colour (blue) but there is not selection for size. Colour is the trait selected and size is a correlated property; more exactly, the blue colour has been favoured by natural selection against the red one, or, from the point of view of our hypothetical Go player, because she can add two stones in each turn, she has an advantage over the other player, who can add only one. As we can see, variational explanation and the concepts of selection-for and selection-of, are not necessarily bounded to a negative, filtering, view.

Our selection game also can reach the same case as Sober's selection toy (Figure 2a) with a little modification. There are blue and red balls again, but they have different sizes the blue ones being bigger than the red ones. In this variation of the game, red and blue balls have the same replication rate. Moreover, the space of the screen is divided by walls or doors placed in different levels. Depending on the level the walls leave wider or narrower gaps. The game starts. There is no difference between the reproductive success of red and blue balls in the first generations (i.e. there is not selection). They can pass through the openings in the first level walls. But, in the sixth generation, the blue balls' population stops growing because it is unable to overcome the second level walls. Meanwhile, red balls also achieve the second level filling completely the screen, increasing their frequency (Figure $2 b$ ). This example is analogous to Sober's one because it establishes a process of filtering individuals where only small red balls are selected, it being the size of the openings of the walls which shows that there is selection for being small and not for being red.

\section{Cui Bono}

The impact produced by an image is hard to remove from our consciences. If we want, as we do, to modify the concept associated to an image by presenting another one, it is necessary to add to the claim an explanation about the pros and cons which are represented and, mostly, who is the beneficiary of this change.

\subsection{Where Fodor and Piattelli-Palmarini got wrong}

Sober's selection toy has become an iconic figure. Consequently, it has also been converted into a perfect target for authors, who have been well 


\begin{tabular}{|l|l|l|l|l|l|}
\hline & & & & \\
\hline
\end{tabular}

\section{Figure 2a}

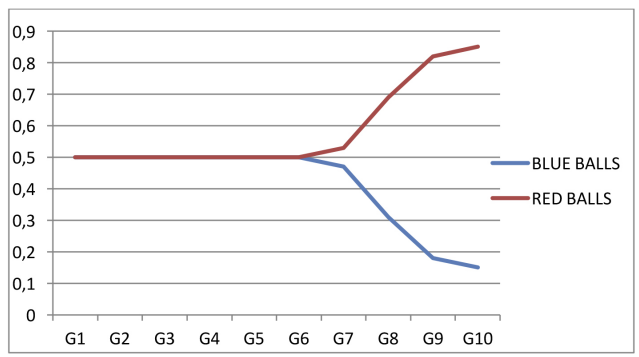

Figure 2b 
or badly informed, to shot at. An example of this is found in the disproportionate attack carried out by Jerry Fodor and Massimo Piattelli-Palmarini (hereafter FPP) in their book What Darwin got wrong ${ }^{19}$. Their vision of evolutionary biology ${ }^{20}$, especially in relation to their anachronistic approach to adaptation ${ }^{21}$, became their form of attack but posed no real challenges, only fireworks. Nevertheless, what we can focus on is in the way they take on Sober's selection toy and check if our selection game is able to resolve those problems that FPP have with the selection toy.

From a simplistic view of natural selection such as a simple filter that acts over existing variation ${ }^{22}$, FPP object not only to the difference between selection-for and selection-of, but natural selection itself. FPP consider natural selection as something "irredeemably flawed" because it is unable to explain why some traits have been selected over others. In other words, FPP claim that given two traits - A and B -, there is no way to discern if there has been selection for $A$ or selection for $B$ in $a$ selective process because both traits are coextensive ${ }^{23}$. Darwin believed it is possible because, in the same way that in an artificial selection process the breeder knows which trait has been selected, natural selection distinguishes between a causally relevant trait and a simply correlated one. At first sight Sober's selection toy solved precisely this problem. The mechanism of the toy itself - which selects size but not colour lets us know which trait has been selected - because of its causal relevance - and which one is correlated. Nevertheless, Sober got it wrong, according to FPP:

But notice that this way of describing what happens is entirely arbitrary even if the mechanism that performs the sorting is exhaustively specified. Sober must be thinking

19 Fodor and Piattelli-Palmarini, 2010.

20 Futuyma, 2010.

21 Luque and Moya, 2014.

22 See Fodor and Piattelli-Palmarini, 2010, 23.

23 FPP seem not to be aware that evolutionary biologists are very conscious of these problems and, as we pointed out in footnote 13, have developed multivariate statistical methods as genetic variance-covariance matrix, selection gradients, fitness surfaces, etc., and even experimental manipulation in order to deal with them (Conner and Hartl, 2004; Fairbairn and Reeve, 2001). 
of the kind of sorting that goes on when you sift flour: the mixed stuff goes into the top of the machine, the good stuff comes out of the bottom of the machine; and what's left behind is the bad stuff. Suppose, however, one thinks not of sifting flour but (for example) of panning for gold. In that case, it's the bad stuff that goes to the bottom and the good stuff that is left as the residuum. What the machine is sorting-for depends on what the prospector had in mind when he did the sorting ${ }^{24}$.

Sober replies that he was aware of such ambiguity and that was the reason why he asks the reader to imagine "that 'the name of the game' is for the ball to get to the bottom"25). Anyway, the goal of the game could be to select the smaller balls (lowest) or the bigger ones (highest). In both cases the toy selects for size and not for colour. However, natural selection is not ambiguous: "there is selection for traits that enhance survival and reproduction, not for traits that do the opposite"26.

Diéguez proposes as a solution an approach more in the way of how natural selection actually works in nature: "Imagine now that a machine destroys all the balls that are above the sieve and another machine makes copies of those which have reached the bottom of the device. In the end, there will not be any big red ball but there will be twice the number of small green balls. Then, is there any doubt about what has been selected?"27.

It is clear that the key to the solution lies in a more realistic way of representing natural selection. Actually, natural selection makes copies (i.e. replication) in nature. Is it possible to apply FPP's criticisms to the selection toy in our selection game? In the first example (Figure 1a), there is no filter consequently the question about which trait has been selected is avoided. On the other hand, there is a sieve in Figure $2 \mathrm{a}$, but it is not ambiguous: red balls pass through the openings on the walls

24 Fodor and Piattelli-Palmarini, 2010, 129 (emphasis in the original).

25 Sober, 2010, 604.

26 Sober, 2010, 605.

27 Diéguez, 2011,348 (my translation). 
because of their (smaller) size, and that is the reason why blue balls do not. Moreover, the replicative dynamic removed the potential ambiguity which appears in the selection toy. Small red balls are selected because they are fitter than the big blue ones (that's shown in their respective population numbers). The red balls' size allows them to pass through the walls of the second level. Replication lets us differentiate, unquestionably, where there is selection-for because it has directionality. There is a unique difference between the balls shown in Figure 1a, i.e., their colour. Therefore if the blue balls are fitter than the red balls (they produce more copies), this is a consequence of their colour. Balls in Figure $2 \mathrm{a}$ have different sizes and colours. Blue balls are as fit as red balls until they arrive to the second level walls. It is obvious that size and not colour is responsible for the increase of red balls fit. The selection game shows that the reproductive success is the most important fact to natural selection. Survival is a necessary condition but is not always enough to produce selection. Reproductive success is a better solution to explain selection and, consequently, a better visual explanation of natural selection which includes replication.

\subsection{Back to the principles}

Our approach to natural selection is closer to the one defended by the fathers of Modern Synthesis than the original one claimed by Darwin. In other words, our result is more Fisherian than Malthusian. We highlight the reproductive success and not the mere survival. In Oscar Wilde's words: "Nothing succeeds like excess"28. The reproductive success can be produced in different ways - survival, mating success, fecundity, etc. but viable selection is just one of them ${ }^{29}$. Sober is aware of it ${ }^{30}$; selection is sometimes akin to tennis and sometimes akin to golf ${ }^{31}$. In the first

28 Endler, 1986, 29. Endler points out the connection with Fisher (1930) using the concept of "excess". His Fundamental Theorem of Natural Selection postulates that there is evolution by natural selection when the average excess of fitness of an allele is positive (increasing in frequency) or negative (decreasing in frequency), where natural selection favours those alleles with positive average excess of fitness (Templeton, 2006).

29 Actually, selection seems to be stronger with the elements related to reproduction (Kingsolver et al., 2001).

30 Sober, 1984. 194-195.

31 Sober, 1984, 17. 
case, scoring implies that the other player does not score; in the second one our score is not affected by the other player.

Brandon ${ }^{32}$ has pointed out that differential reproduction is needed for selection because a selection process in viability can be counteracted in another stage of the life cycle - the breaking down of an organism's life cycle comes from the necessity of visualize theses counterbalances. Sober's selection toy can only explain viability. Selection in viability, where some individuals survive and some others die, is shown by the reproductive success of all of them (those which survive will leave offspring, those which die will not); but, if there is also selection in fecundity, survival will be not enough. In both cases, differential reproduction shows which types are the fittest ${ }^{33}$.

Nevertheless, we must point out a selection toy's virtue. It clarifies the difference between phenotypic selection and evolution by natural selection. This difference, which comes from Quantitative Genetic by analogy with artificial selection, establishes that a selection process appears within generations while evolution by natural selection is a process across generations as a response to selection within generations and dependent on hereditary component ${ }^{34}$. This distinction does not have full consensus. Some other evolutionary biologists ${ }^{35}$ include inheritance in their natural selection definition. Michod especially insists on defending the inclusion of it because he believes that it is not so easy to distin-

32 Brandon, 2008.

33 Bouchard (2008) points out that some biological systems - certain colonies and symbionts - resist this replicative picture because it is not easy to distinguish different organisms inside these systems. In order to overcome this problem, Bouchard argues for focusing on difference persistence, that fitness is not only about reproductive success but can be re-stated as a propensity of the organism to persist longer. Nevertheless, biologists have resources in addressing some of these particular systems. For example, they adopt a gene-eye view for eusocial insects, measuring fitness as the differential reproductive success of different alleles. On the other hand, my replicative approach applies to the rest of biological systems (prokaryotes and eukaryotes), and those particulars systems stressed by Bouchard (for instance, fungus-termites colonies) are the evolutionary product of millions of years of replicative organisms.

34 See Brandon, 1990; Fairbain and Reeve, 2001; Futuyma, 2013. The breeder's equation $R=h^{2} S$ shows this distinction, where response to selection, $R$, is equal to heritability, $h^{2}$, multiply by the selection differential, $S$, which represents the mean phenotype of the selected parents minus the overall mean of the total population.

35 Endler, 1986, 4; Ridley, 1996, 71-72; Michod, 1999, 17. 
guish between the selection of phenotypes and response to selection, particularly when there is selection simultaneously at different levels of the organization.

Heredity is included in our selection games (individuals who replicate themselves without error through cloning) in order to show selection directionality and avoid misunderstandings produced by the inheritance transmission. A conceptualization always implies a trade-off. The author should assess which are the advantages and disadvantages of it. I consider that our image of selection, shown by the selection game, offers more benefits than losses. Its principal advantage is the recovering of reproduction as a central element in the selection process. Moreover, any interesting question about natural selection - its roles as a causal force in maintenance and spreading traits; as a force which creates adaptations; its interaction with other evolutionary forces; its role in the increase of biological complexity, etc. - are evolutionary questions and therefore involve an evolutionary conceptualization where inheritance has a fundamental role.

Because, in the end, as one of the main defenders of selection within and across generation distinction claims: "Although we adopt the phenotypic perspective, we will almost always discuss natural selection among heritable phenotypes because selection seldom has a lasting evolutionary effect unless there is inheritance" 36 .

\subsection{Fitness and the causes of evolution}

Fitness is a key concept in evolution and, specially, for evolution by natural selection. As explained above in section 2, fitness differences are typically required in order to produce evolution by natural selection. Nevertheless, a properly and unique definition of fitness has not yet developed, and a huge and overwhelming literature has been written in the last fifty years on this topic ${ }^{37}$. Usually biologists define fitness as the ability of an organism to survive and reproduce in a specific environment ${ }^{38}$, and this is a comparative notion - how good or bad is an organ-

36 Futuyma 2013, 286.

37 For a recent overview, see Ramsey and Pence 2013.

38 Conner and Hartl, 2004; Orr, 2009. 
ism compared to other organism of the same population. This intuitive definition contrasts with the great variety of fitness measures developed by researchers. This double nature of fitness has made that some authors cast doubt on the causal efficacy of fitness, and by extension on the causal efficacy of natural selection ${ }^{39}$. If we take fitness as a measure of the change of trait distribution, usually by counting organisms' offspring, then obviously fitness does not play a causal role. As explained in section 1.1, Sober also argued that the overall fitness of an organism is not causally efficacious but nonetheless natural selection is, due to the selection-for concept. Recently ${ }^{40}$, Sober claims that variation in fitness plays a causal role promoting evolution, although variation in fitness is a property of the population, a propensity the population has to change traits distribution over time. Some could argue that Sober is defending two distinct causes. Nevertheless, variation in fitness and selection-for are not distinct causes, in the way selection and migration are distinct. But both notions describe causes ${ }^{41}$. Variation in fitness describes a causal process at the population level but it does not tell us what trait (or traits) exactly is (are) under selection-for, or, in other words, what exactly kind of property is. Then, selection-for would be more specific, indicating the exact trait (or traits) which is under selection-for.

This double nature of fitness, an organism's ability and a measure, triggered what has come to be known as the dynamic vs. statistical debate ${ }^{42}$. Since Darwin's times until our current days, evolutionary theory has been conceptualized as a causal theory. As the evolutionary literature talk about evolutionary forces acting on a population, Sober proposed an analogy with Newtonian mechanics, where evolutionary theory is a theory of forces because, in the same way that different forces of Newtonian mechanics cause changes in the movement of bodies, evolutionary forces cause changes in trait frequencies. As a result, selection, drift, mutation

39 Walsh et al, 2002; Matthen and Ariew, 2002.

40 Sober, 2013.

41 There is an analogy here between "mental state M" and "neutral state N". Both can describe the causes of a given behaviour, but they are not independent of each other if $M$ supervenes on $\mathrm{N}$.

42 Walsh et al, 2002; Matthen and Ariew, 2002; Bouchard and Rosenberg, 2004; Millstein, 2006. See Otsuka 2016 a for a recent review. 
and migration would be the main forces or causes of evolution. This is known as the dynamic view. Nevertheless, the appropriateness of this view has been challenged by the statistical view, where the evolutionary process and its parts (selection, drift, etc.) are mere statistical outcomes, inseparable from each other. The so-called evolutionary forces should be conceptualized as statistical population-level tendencies, abandoning any causal role for them.

All these debates go beyond the scope of this paper, so I will not go into detail here. I support the view developed by several dynamic authors - also called causalists - that fitness and natural selection are causally efficacious ${ }^{43}$. My selection game shows the comparative nature of fitness and natural selection, since we have two different types competing in the same environment, and the differences in reproductive success - a way of measuring fitness - are due to their different abilities to reproduce. In the first example (Figure 1) the differences in reproductive success are due to the ability of blue balls to replicate twice faster than red ones - we can call it a fertility trait. In the second example (Figure 2) the differences in reproductive success are due to the ability of red balls to pass through the second level of walls - due to a particular trait, their size. These two games show a causal process where different entities with different abilities to survive and reproduce have different reproductive success. This causal process is called natural selection. These two examples easily fit, not only on experimental biology carried out with microorganisms, but also into a recent theoretical work developed by Günter Wagner ${ }^{44}$ who devises a pairwise competition test where, in order to amplify the effects of selection, the competition is run over many generations. This allows him to develop a ratio scale measure of fitness and derive fundamental population genetics equations. Our selection game also shows that, contrary to the claim of the statistical view, one can defend the causal status of natural selection without appealing to an extra or third entity (a tertium quid). In order to have evolution by natural selection you only need different entities with different abilities to survive and reproduce. Nothing more, nothing else.

43 Bouchard and Rosenberg, 2004; Millstein, 2006; Wagner, 2010; Otsuka, 2016b.

44 Wagner, 2010. 


\subsection{Creative and Negative view revisited}

In this article I have criticized some fundamental claims of the Negative View - especially that selection is a force which only removes or filters existent variants. One might think that we are favouring the Creative View. That is not completely right. Firstly, most of the Creative View defenders ${ }^{45}$ assume the Malthusian view of natural selection, according to which it is like a filter which simply eliminates organisms. It would seem that it is a good reason to explain why it has been so difficult to develop a positive view of selection and why it seems so counterintuitive, becoming the target of critics like the ones defended in this paper. Secondly, the defenders of the Negative View could agree with the selection game because there are no creative elements in it either, that is essential elements as cumulative steps. Indeed, the selection game does not represent a creative selection but the simple change of frequency of extant variants. What we were facing was a distributive role, not an origin role, because there is no mutation or other mechanism that provides variation into the population. Assuming this, the defenders of the Creative View might find some loopholes in the selection game:

a) Notions such as positive selection, the spread of a new variant in the population by natural selection, are vindicated by the selection game instead of a simple maintenance of a variant by removing new deleterious mutations - known as negative or purifying selection ${ }^{46}$. Razetto-Barry and Frick ${ }^{47}$ argue that positive selection could be creative because it acts over long periods of time, increasing the probability of the emergence of new advantageous mutations and allowing its accumulation.

b) The selection game also defends replication. Martínez and Moya ${ }^{48}$ defend that natural selection has a creative role in the morphology of organisms because its affect on future genetic materials due to it imposing an evolutionary direction. This future genetic material is that which will create the individuals. This feedback process of upward and downward causation is only possible through several steps, where replication

\footnotetext{
45 Ayala, 1970; Neander, 1995; Nanay, 2005.

46 Charlesworth and Charlesworth, 2010.

47 Razetto-Barry and Frick, 2011.

48 Martínez and Moya, 2011.
} 
and heredity are crucial in order to preserve the timeline (heredity) and the achievement of new genetic material, channelling it, and creating new individuals (replication).

Furthermore, this link between the Creative View and our replicative approach is supported by empirical research. Lenski and colleagues ${ }^{49}$ have developed a long-term evolution experiment - over 50000 generations - started in 1988 with twelve identical populations of Escherichia coli from the same clone. These populations have evolved in a medium of glucose as the limiting resource, with identical environmental conditions, and every day a small sample of each population is transfered to a new flask, starting a new replication cycle. In addition, a sample of each population has been frozen every 500 generations, remaining these samples viable. In these conditions, natural selection favoured those individuals capable of using glucose in a more efficient way. Therefore, these individuals expanded faster than the others, and monopolize their linage over few generations. When a new mutant appeared using glucose in a more efficient way, it monopolizes the linage, and so on ${ }^{50}$. Lenski and colleagues were able to compare bacteria's fitness from different generations, in a pairwise competition test, where evolved or "modern" bacteria showed faster growth rates than ancestral ones. Moreover, the medium also contained citrate, but Escherichia coli cannot exploit citrate. Nevertheless, one population was able to use citrate as a carbon and energy source. The explanation for the appearance of this very rare variant required more preceding mutations in very specific steps, maintained each one in the population by the action of natural selection.

All this long-term experiment and their correlates are based on the replicative approach, on observe the action of natural selection (combined with mutation and genetic drift) on organisms replicating over long periods of time. Here, selection is not pictured as a filter, but as an amplifier. These microbiological experiments are a good example for the view that I have developed in this paper.

49 See Blount et al., 2008; and Blount et al., 2012.

50 Researchers also observed various examples of parallel evolution: "All evolved higher maximum growth rates on glucose, shorter lag phases upon transfer into fresh medium, reduced peak population densities, and larger average cell sizes relative to their ancestor" (Blount et al. 2008, 7899). 


\section{Conclusion}

I have argued that the image of natural selection given by Sober and other defenders of the Negative View is problematic. The prototypical representation of it as a filter, in the selection toy and in the variational explanation, implies the loss of essential elements of the selection as replication. These problems were visible when we realized that the selection toy was unable to distinguish between selection-of and selection-for in indisputable cases of natural selection. This has motivated the development of a selection game which can provide a more accurate view of selection just like it happens in nature. Moreover, it can distinguish between selection-of and selection-for in all those cases where the selection toy was unable. Sober's toy is useful and advantageous because it is open to multiple readings and extensions. Nevertheless its motionlessness and also its analogic nature (it is a game which needs to be shaken when handled) has the charm but also the limits of something vintage. My animation adds dynamics to the image as well as a temporal arrow which removes any possible misunderstanding about the direction of the filtering process. I think that the selection game gives a more realistic, and consequently less problematic, view of natural selection. It removes any possible ambiguity because it is more positive and Fisherian.

\section{Acknowledgments}

Thanks to Andrés Moya, Valeriano Iranzo, Silvia Martínez, Antonio Diéguez, and an anonymous referee for useful comments on an earlier version of this paper. Special thanks to Alejandro Martínez, who developed the selection game's computer programme, capturing faithfully my original idea. This work was funded by the Generalitat Valenciana (Prometeo research program - reference: II/2014/065).

\section{References}

Ayala, F., 1970, Teleological explanations in evolutionary biology. Philosophy of Science, 37, pp. 1-15.

Birch, J., 2012, The negative view of natural selection. Studies in History and Philosophy of Biological and Biomedical Sciences, 43, pp. 569-573. 
Blount ZD, Borland CZ, Lenski RE, 2008, Historical contingency and the evolution of a key innovation in an experimental population of Escherichia coli. PNAS, 105 (23), pp. 7899-7906.

Blount ZD, Barrick JE, Davidson CJ, Lenski RE , 2012, Genomic analysis of a key innovation in an experimental Escherichia coli population. Nature, 489, pp. 513-517.

Bouchard, F., 2008, Causal processes, fitness, and the differential persistence of lineages. Philosophy of Science, 75(5), pp. 560-570.

Bouchard, F. and Rosenberg, A., 2004, Fitness, probability and the principles of natural selection. British Journal for the Philosophy of Science, 55, pp. 693-712.

Brandon, R., 1990, Adaptation and environment. Princeton, Princeton University Press.

--- 2008, Natural selection. In: Stanford Encyclopedia of Philosophy June 2008). http://plato.stanford.edu/entries/natural-selection/. Accessed 18 February 2016.

Charlesworth, B. and Charlesworth D., 2010, Elements of Evolutionary Genetics. Colorado, Roberts and Company Publishers.

Conner, J.K. and Hartl, D.L., 2004, A Primer of Ecological Genetics. Sunderland, Sinauer.

Darwin, C., 1859, The Origin of Species. London, John Murray.

De Vries, H., 1904, Species and Varieties. Their Origin by Mutation, Chicago, Open Court 1904.

--- 1909, Variation. In: Darwin and Modern Science: Essays in commemoration of the century of the birth of Charles Darwin and of the fiftieth anniversary of the publication of the Origin of the Species, Cambridge, Cambridge University Press, pp. 66-84.

Diéguez, A., 2011, Alguien, en efecto, ha debido de malinterpretar algo: el desafío de Fodor y Piattelli-Palmarini al Darwinismo. Ludus Vitalis, 19, pp. 341-357.

Elena, S. and Lenski, R., 2003, Evolution experiments with microorganisms: the dynamics and genetic bases of adaptation. Nature Reviews Genetics, 4, pp. 457-469 
Endler, J., 1986. Natural Selection in the Wild. Princeton, Princeton University Press.

Fairbain, D.J., and Reeve, J.P., 2001, Natural Selection. In: Evolutionary Ecology. Concepts and Case Studies, Oxford, Oxford University Press, pp. 29-43.

Fisher, R., 1930, The Genetical Theory of Natural Selection, Oxford: Clarendon Press.

Fodor, J. and Piattelli-Palmarini, M., 2010, What Darwin Got Wrong, London, Profile Books.

Futuyma, D., 2010, Two Critics Without a Clue. Science, 328(5979), 692-693.

--- 2013, Evolution (third edition). Sunderland: Sinnauer.

Gillespie, J., 2004, Population Genetics. A concise guide (second edition). Baltimore, The John Hopkins University Press.

Godfrey-Smith, P., 2009, Darwinian Populations and Natural Selection, New York, Oxford University Press.

Gould, S.J., 2002, The Structure of Evolutionary Theory, Cambridge, Harvard University Press.

Kingsolver, J.G., Hoekstra, H.E., Hoekstra, J.M., Berrigan, D., Vignieri, S.N., Hill, C.E., Hoang, A., Gibert P. and Beerli P., 2001, The Strength of Phenotypic Selection in Natural Populations. The American Naturalist, 157 , pp. 245-261.

Lande, R. and Arnold, S., 1983, The measurement of selection on correlated characters. Evolution, 37, pp. 1210-1226.

Lewontin, R., 1970, The Units of Selection. Annual Review of Ecology and Systematics, 1, pp. 1-18.

--- 1983, Darwin's Revolution. In New York Review of Books, June 16.

Luque, V.J. and Moya, A., 2014, Between the Seventeenth and Twenty-First Centuries. Teorema, 38(2), pp. 199-210.

Martínez, M. and Moya, A., 2011, Natural selection and Multi-level causation. Philosophy and Theory in Biology, 3. http://quod.lib.umich. edu/p/ptb/6959004.0003.002?view=text;rgn=main. Accessed 20 February 2016. 
Matthen, M. and Ariew, A., 2002, Two ways of thinking about fitness and natural selection, Journal of Philosophy, 99(2), pp. 55-83.

Michod, R., 1999, Darwinian Dynamics, Princeton, Princeton University Press.

Millstein, R., 2006, Natural selection as a population-level causal process. British Journal for the Philosophy of Science, 57(4), pp. 627-653.

Nanay, B., 2005, Can cumulative selection explain adaptation?, Philosophy of Science, 72, pp. 1099-1104.

Neander, K., 1995, Pruning the tree of life. British Journal for the Philosophy of Science, 46, pp. 59-80.

Nowak, M., 2006, Evolutionary Dynamics, Cambridge: Harvard University Press.

Orr, H. A., 2009, Fitness and its role in evolutionary genetics", Nature Reviews, 10, pp. 531-539.

Otsuka, J., 2016a, A critical review of the statisticalist debate, Biology and Philosophy, 31 (4), pp. 459-482.

--- 2016b, Causal Foundations of Evolutionary Genetics, The British Journal for the Philosophy of Science, 67 (1), pp. 247-269.

Ramsey, G. and Pence, $\mathrm{CH}, 2013$, Fitness: Philosophical Problems, In: eLS. John Wiley \& Sons, Ltd: Chichester. DOI: 10.1002/9780470015902. a0003443.pub2

Razeto-Barry, P., and Frick, R., 2011, Probabilistic causation and the explanatory role of natural selection. Studies in History and Philosophy of Biological and Biomedical Sciences, 42, pp. 344-355.

Ridley, M., 1996, Evolution (second edition), Oxford, Blackwell.

Sober, E., 1984, The Nature of Selection, Chicago, University of Chicago Press.

--- 2010, Natural Selection, Causality and Laws: What Fodor and Piattelli- Palmarini Got Wrong. Philosophy of Science, 77, pp. 594-607.

--- 2013, Trait Fitness is not a Propensity, but Fitness Variation Is, Studies in History and Philosophy of the Biological and Biomedical Sciences, 44, pp. 336-341. 
Templeton, A., 2006, Population Genetics and Microevolutionary Theory, New Jersey, John Wiley \& Sons.

Vervust, B., Grbac, I. and Van Damme, R., 2007, Differences in morphology, performance and behaviour between recently diverged populations of Podarcis sicula mirror differences in predation pressure. Oikos, 116 , pp. 1343-1352.

Wagner, G., 2010, The measurement theory of fitness. Evolution, 64(5), pp. 1358-1376.

Walsh D.M., Lewens T., Ariew, A. (2002) The trials of life: natural selection and random drift. Philos Sci 69(3), pp. 452-473. 